\title{
Optimisation de l'alimentation des machines à réluctance variable polyphasées avec inductances mutuelles
}

\author{
X. Tian et C. Glaize \\ L.E.SI.R.-E.N.S. de Cachan, 61 av. du Président-Wilson, 94230 Cachan, France
}

(Reçu le 30 juillet 1987, révisé le 28 octobre 1987, accepté le 2 novembre 1987)

\begin{abstract}
Résumé. - Cet article a pour objet une étude des modes d'alimentation des machines à réluctance variable à phases magnétiquement couplées. Nous cherchons les formes optimales de courant d'alimentation en minimisant le facteur de dimensionnement du convertisseur. Nous comparons ensuite les différents modes d'alimentation des machines à réluctance variable triphasées et nous étudions les influences de la fréquence d'alimentation, des connexions électriques, de l'excitation et des caractéristiques des machines sur le facteur de dimensionnement et l'ondulation du couple.
\end{abstract}

\begin{abstract}
This paper deals with the feeding modes of reluctance machines having magnetic coupling between the phases. We propose to determine the optimal waveforms of supply current by minimizing the sizepower ratio of converters. A comparison is then made between different feeding modes for three-phase reluctance machines. The influences of the feeding frequency, the electric connections, the excitation and the machine parameters on the size-power ratio and the torque ripple are discussed.
\end{abstract}

\section{Introduction.}

Par rapport aux autres machines classiques, les machines à réluctance variable (M.R.V.) présentent la particularité de ne pas posséder de conducteur au rotor. Cette simplicité leur confère une grande robustesse et leur permet un fonctionnement à grande vitesse. Grâce à de nouvelles structures et au développement de l'électronique de puissance, ces machines sont de plus en plus utilisées dans les systèmes à vitesse variable. Pour améliorer les performances de la machine et diminuer le dimensionnement de convertisseur, il est intéressant de chercher les formes optimales d'onde d'alimentation. Car avec différentes structures de convertisseur et de commande, on peut, en principe, réaliser toutes les formes d'onde de courant d'alimentation. C'est dans ce but que travaillent des chercheurs du L.E.SI.R. (E.N.S. de Cachan). Une première recherche de formes optimales du courant d'alimentation a été effectuée en minimisant les pertes Joule et le courant maximal à couple moyen donné [1]. Ensuite, par la prise en compte de la tension maximale qui joue un rôle important dans le dimensionnement du convertisseur, une deuxième étude a été faite en minimisant le facteur de dimensionnement [2]. Ces travaux ont essentiellement porté sur des M.R.V. monophasées et multimonophasées, ces dernières ne possèdent pas de couplage magnétique entre phases. Le présent travail se propose de chercher les formes optimales d'alimentation pour des M.R.V. triphasées avec inductances mutuelles et étudie les influences des mutuelles et des connexions électriques des phases.

\section{Modèle de la M.R.V. triphasée.}

Nous considérons la structure élémentaire des M.R.V. triphasées représentée sur la figure 1 , où $\theta$ désigne la position angulaire du rotor par rapport au stator.

Les tensions aux bornes des enroulements peuvent être calculées par :

$$
v_{k}=R i_{k}+\frac{\mathrm{d} \Phi_{k}}{\mathrm{~d} t} \quad k=1,2,3 .
$$

Le flux dans chaque enroulement est une fonction de tous les courants et de la position du rotor $\theta$ :

$$
\Phi_{k}=\Phi_{k}\left(i_{1}, i_{2}, i_{3}, \theta\right) \quad k=1,2,3 .
$$




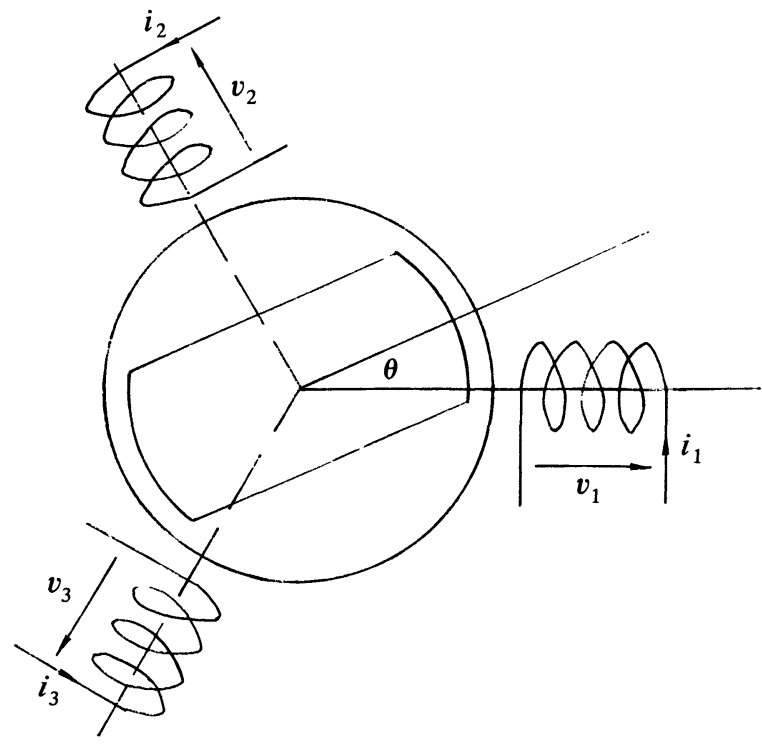

Fig. 1. - Structure de la M.R.V. triphasée.

[Structure of a three-phase reluctance machine.]

Le couple électromagnétique peut s'écrire en fonction du flux et du courant à partir de l'expression de la coénergie magnétique $W^{\prime}$ :

$$
c=\frac{\partial W^{\prime}}{\partial \theta}=\int \sum_{k=1}^{3} \frac{\partial \Phi_{k}}{\partial \theta} \mathrm{d} i_{k} .
$$

Ces trois équations de base permettent au moins d'effectuer deux types de calcul essentiels dans une simulation :

- la forme du courant étant donnée, on cherche la tension et le couple instantanés.

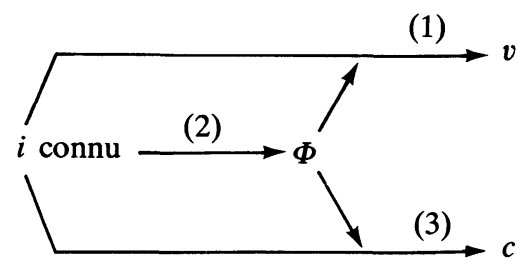

- la forme de la tension étant donnée, on cherche le courant et le couple instantanés.

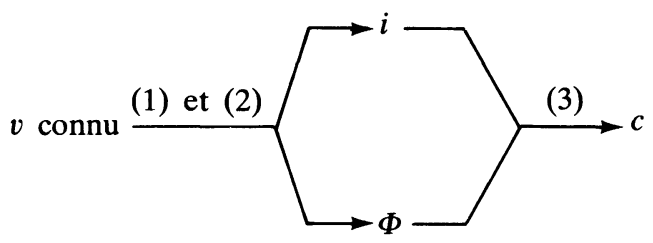

Pour simplifier les calculs, nous imposons deux hypothèses sur le modèle magnétique de la machine (Eq. (2)) : pour une position $\theta$ donnée, le flux est une fonction linéaire du courant $i$; pour un courant $i$ donné, on ne considère que le fondamental du flux en fonction de la position $\boldsymbol{\theta}$.
Nous avons ainsi un modèle linéaire et sinusoïdal. L'équation (2) peut alors se développer :

$$
\left(\begin{array}{l}
\Phi_{1} \\
\Phi_{2} \\
\Phi_{3}
\end{array}\right)=\left(\begin{array}{lll}
l_{11} & l_{12} & l_{13} \\
l_{12} & l_{22} & l_{23} \\
l_{13} & l_{23} & l_{33}
\end{array}\right) \quad\left(\begin{array}{l}
i_{1} \\
i_{2} \\
i_{3}
\end{array}\right)
$$

où :

$$
\left\{\begin{array}{l}
l_{11}=L+\Delta L \cos 2 \theta \\
l_{22}=L+\Delta L \cos 2(\theta-2 \pi / 3) \\
l_{33}=L+\Delta L \cos 2(\theta-4 \pi / 3) \\
l_{12}=M+\Delta M \cos 2(\theta-4 \pi / 3) \\
l_{23}=M+\Delta M \cos 2 \theta \\
l_{13}=M+\Delta M \cos 2(\theta-2 \pi / 3)
\end{array} .\right.
$$

Les quatre coefficients $L, \Delta L, M$ et $\Delta M$ qui déterminent entièrement le modèle simplifié doivent être choisis selon les caractéristiques de la machine étudiée. Quand $M=\Delta M=0$, le modèle représente les machines sans couplage magnétique entre phases (machines multimonophasées). Quand $\Delta M=\Delta L$, le modèle représente les machines avec la répartition sinusoïdale des f.m.m. des enroulements statoriques (M.R.V. classiques). Si $M$ et $\Delta M$ prennent d'autres valeurs, le modèle peut approximativement représenter toutes les M.R.V. triphasées avec mutuelles (par exemple, certaines machines avec enroulements concentrés).

\section{Nouveau facteur de dimensionnement.}

En électrotechnique classique (charges linéaires alimentées en sinusoïdal), le facteur de dimensionnement est classiquement défini par :

$$
\delta_{c}=\frac{v_{\mathrm{eff}} i_{\mathrm{eff}}}{P}=\frac{1}{\cos \Phi}
$$

où $v_{\text {eff }}$ et $i_{\text {eff }}$ sont les valeurs efficaces des tensions et des courants ; $P$ est la puissance active et $\cos \Phi$ est le facteur de puissance.

Dans les systèmes alimentés par des convertisseurs statiques, les tensions et les courants ne sont plus sinusoïdaux. Ce facteur de dimensionnement n'est plus très significatif. Un convertisseur statique est normalement dimensionné par le nombre de composants semiconducteurs, le dimensionnement de chaque composant, la complexité des circuits auxiliaires et des circuits de commande, etc. Pour une structure donnée, nous voulons minimiser les contraintes sur les composants semiconducteurs du convertisseur. On peut donc définir un nouveau facteur de dimensionnement $\delta$ par analogie avec $\delta_{c}$ en remplaçant les valeurs efficaces par les valeurs crêtes qui caractérisent la plupart de composants semiconducteurs (transistors, GTO, ...) [2].

$$
\delta=\frac{v_{\max } i_{\max }}{P}
$$


Le facteur de dimensionnement ainsi défini se détermine entièrement à partir des formes d'onde de tension et de courant de la machine. Une comparaison des différentes structures de convertisseur doit aussi prendre en compte le nombre de composants dans le convertisseur [2]. Dans un premier temps, nous allons optimiser les formes d'onde de courant d'alimentation en minimisant le facteur de dimensionnement. Le nombre de composants n'intervient que dans le choix final de la structure des convertisseurs.

\section{Remarques sur l'alimentation des M.R.V.}

4.1 FréQuence D'Alimentation. - Rappelons que pour une machine linéaire sans couplage magnétique, le couple est proportionnel au carré du courant $\left(c=1 / 2 i^{2} \mathrm{~d} l / \mathrm{d} \theta\right)$, les deux formes de courant de la figure 2 créent donc exactement le même couple $[1,2]$.
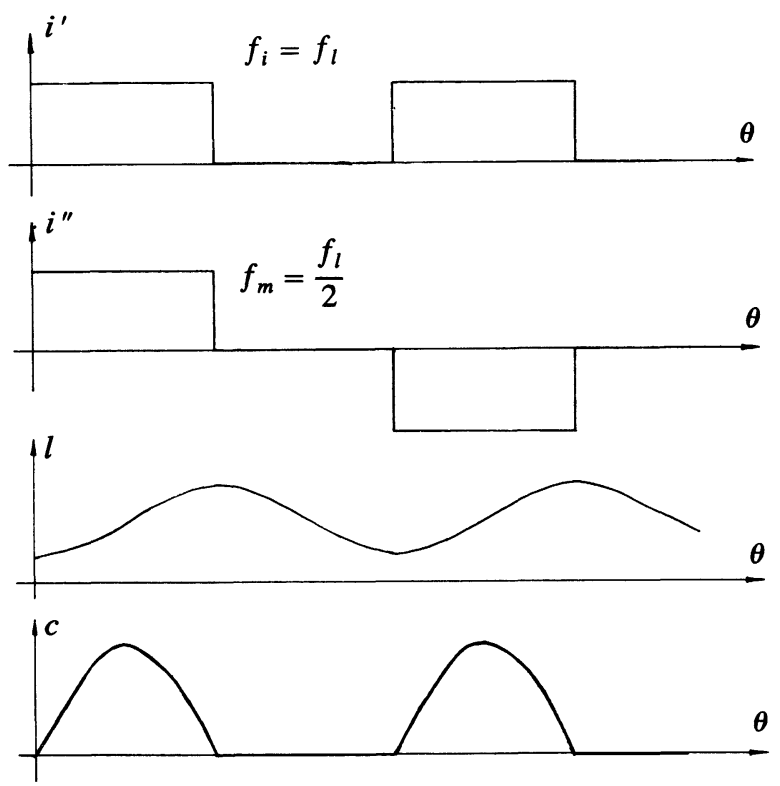

Fig. 2. - Deux fréquences d'alimentation.

[Two feeding frequencies.]

La fréquence du courant $i^{\prime}$ est identique à celle de l'inductance $l$, alors que la fréquence du courant $i^{\prime \prime}$ est égale à la moitié de celle de l'inductance $l$. Deux fréquences fondamentales d'alimentation sont donc utilisables pour les machines sans mutuelle. Nous étudierons le comportement des M.R.V. triphasées pour ces deux fréquences d'alimentation.

\subsection{CONNEXIONS ÉLECTRIQUES DES PHASES. -} Pour éviter une confusion, on emploie les termes couplage magnétique et connexion électrique. Les enroulements des trois phases peuvent être connec-

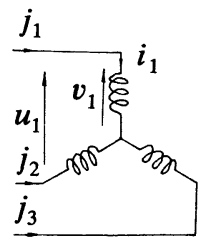

(a)

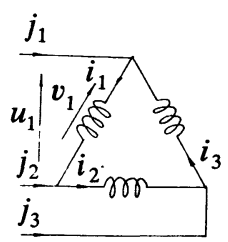

(b)

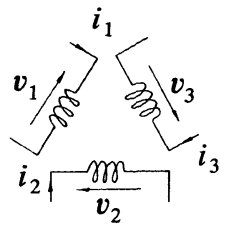

(c)
Fig. 3. - Connexions électriques des phases.

[Electrical phase connections.]

tées en étoile, en triangle ou sans connexion électrique (tri-monophasées) (Fig. 3).

L'expression du facteur de dimensionnement est différente pour chaque connexion car ce sont la tension composée et le courant de ligne qui agissent directement sur le convertisseur. De plus, chaque type de connexion impose des relations entre les divers courants et/ou tensions (contraintes d'optimisation). Le facteur de dimensionnement $\delta$ a alors pour expression :

- en étoile sans neutre (Fig. 3a)

relations : $\quad\left\{\begin{array}{l}i_{1}+i_{2}+i_{3}=0 \\ u_{1}+u_{2}+u_{3}=0\end{array}\right.$

- en triangle (Fig. 3b)

relations : $\quad\left\{\begin{array}{l}v_{1}+v_{2}+v_{3}=0 \\ j_{1}+j_{2}+j_{3}=0\end{array}\right.$

- tri-monophasés (Fig. 3c)

$$
\delta=i_{\max } v_{\max } / P
$$

aucune relation imposée.

Nous étudierons les influences de ces différentes connexions sur le facteur de dimensionnement.

4.3 STRuCtures DES CONVERTISSEURS. - Nous donnons sur la figure 4 trois exemples de structure de convertisseurs pour l'alimentation des M.R.V. triphasées. Les structures (a) et (b) s'emploient dans le cas des machines tri-monophasées. La structure (c) est utilisée pour les connexions en étoile ou en triangle.

Dans le circuit (a), le courant dans une phase ne peut passer que dans un seul sens : c'est un convertisseur unidirectionnel en courant. Dans le circuit (b), le courant passe dans les deux sens : c'est donc un convertisseur bidirectionnel en courant. Pour les connexions en triangle et en étoile (circuit (c)), le convertisseur doit nécessairement être bidirectionnel en courant en raison de la symétrie des phases. Nous pouvons constater que le circuit (b) exige deux fois 


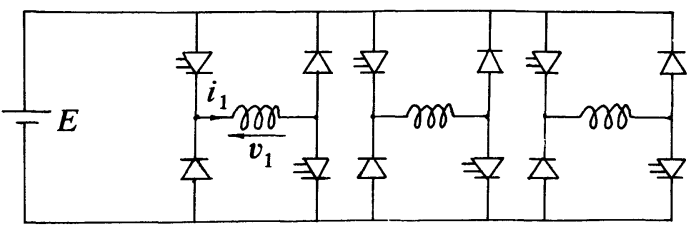

(a)

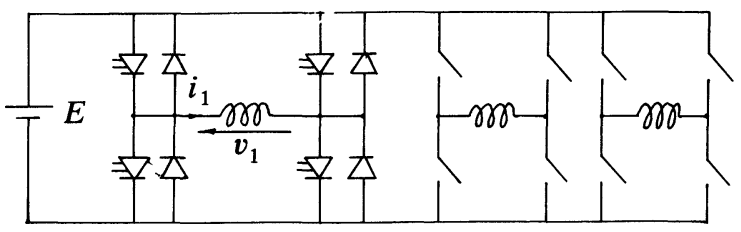

(b)

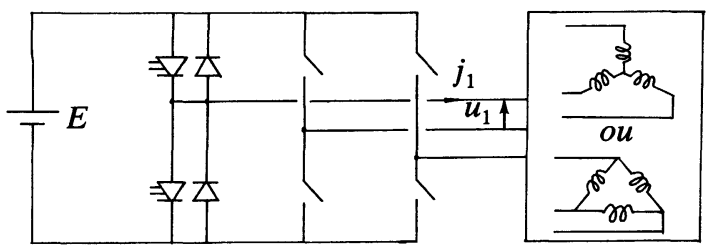

(c)

Fig. 4. - Structures des convertisseurs.

[Converter structures.]

plus de composants semiconducteurs que les circuits (a) et (c).

4.4 ExCitATION. - Il peut être intéressant de réaliser une M.R.V. dite «excitée» $[1,2]$. Chaque enroulement de phase de la machine est dans ce cas divisé en deux parties : un enroulement alternatif (alimentation) et un enroulement continu (excitation). Nous supposons que ces deux enroulements sont magnétiquement parfaitement couplés.

$A$ priori, les enroulements d'excitation des trois phases peuvent être reliés en série ou en parallèle et être alimentés par une source de tension ou une source de courant (Fig. 5). Mais compte-tenu du couplage parfait des enroulements continus et des enroulements alternatifs, la mise en parallèle n'est

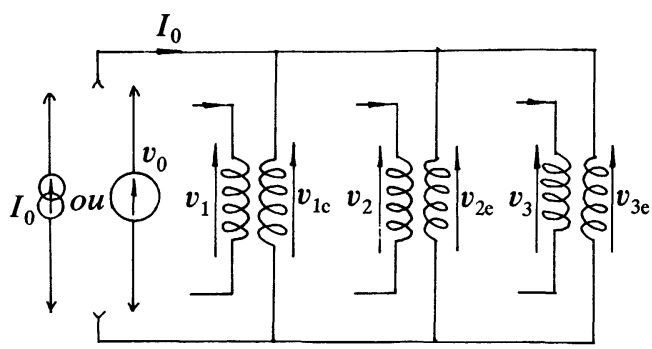

Fig. 5. - Les connexions des enroulements d'excitation.

[Connection of excitation windings.] pas réalisable quand les chutes de tension résistives sont négligeables : elle imposerait l'égalité des trois tensions de phase et donc des variations des trois flux.

Si les enroulements principaux sont alimentés en tension, on ne peut pas aussi imposer une tension aux bornes des enroulements d'excitation, car cette tension présente déjà la forme de tension des enroulements principaux pour une chute de tension résistive négligeable. Si les enroulements principaux sont alimentés en courant, les enroulements d'excitation peuvent alors être alimentés en tension ou en courant. Pour être général, nous supposons par la suite que les enroulements d'excitation sont mis en série et alimentés par une source continue de courant.

Pour la machine excitée, le facteur de dimensionnement doit tenir compte du « coût » du courant d'excitation. Un coefficient $\alpha$ appelé le facteur de pondération du coût du courant d'excitation par rapport au courant alternatif a ainsi été introduit [1, 2]. L'expression du facteur de dimensionnement avec excitation devient :

$$
\delta=\frac{\left(i_{\max }+\alpha I_{0}\right) v_{\max }}{P}
$$

où $I_{0}$ est le courant continu d'excitation ramené aux enroulements alternatifs par équivalence des ampères-tours. Le convertisseur pour l'excitation peut être réalisé par un simple redresseur. Il est en général moins « coûteux » que le convertisseur pour l'alimentation alternative qui est souvent constitué par un redresseur et un onduleur. Il a été montré que l'excitation perdrait tout intérêt si la valeur de $\alpha$ est supérieure à 0,5 [9]. Dans un cas général, il semble que 0,2 est une valeur réaliste de $\alpha[10]$.

\section{Optimisation.}

Les formes d'onde de courant d'alimentation qui minimisent le facteur de dimensionnement sont déterminées de la manière suivante :

Les fonctions sont tout d'abord discrétisées en $N$

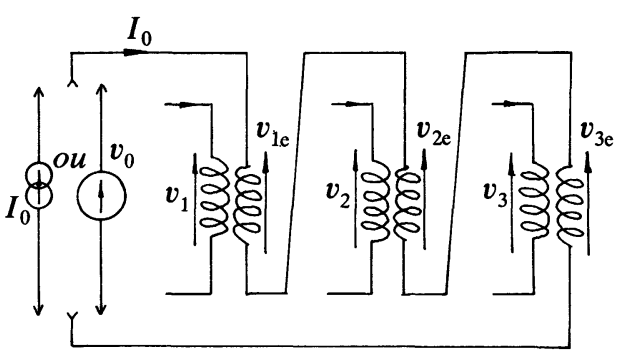


échantillons sur une période. Le problème difficile de l'optimisation de la fonction $i$ devient ainsi une optimisation de $N$ variables (Fig. 6). Le courant est initialisé pour une puissance donnée. On décrémente ou incrémente ensuite certains échantillons tirés au hasard (par exemple $\mathrm{K}$ et $\mathrm{J}$ ) et on calcule le nouveau facteur de dimensionnement. Si le facteur de dimensionnement est amélioré on remplace les échantillons par les nouvelles valeurs. Statistiquement, quand le nombre de tests augmente, les $N$ échantillons seront testés et le courant tendra vers la forme optimale d'alimentation. Il faut cependant vérifier les relations électriques (contraintes d'optimisation) imposées par les connexions, la structure des convertisseurs et la symétrie des phases.

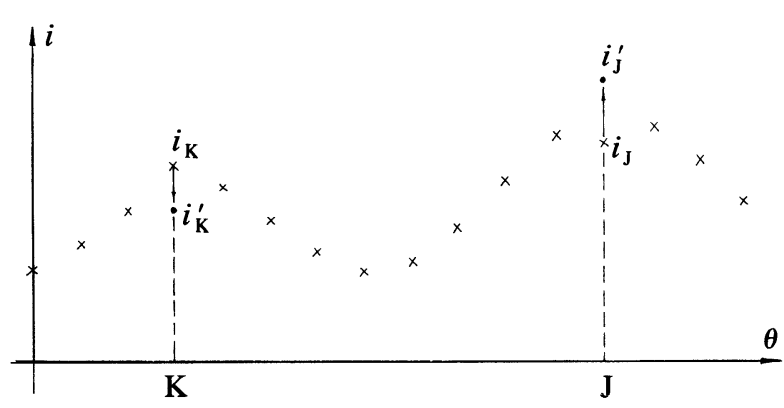

Fig. 6. - Discrétisation d'une fonction.

[Function discretisation.]

Comme les différentes connexions imposent des contraintes électriques différentes et des expressions différentes du facteur de dimensionnement (Sect. 4.2), nous devons écrire un programme d'optimisation pour chaque connexion. Les calculs d'optimisation de chaque programme s'effectuent pour les différentes caractéristiques de M.R.V. triphasées. Par souci de diminuer le nombre de paramètres d'étude, nous négligeons la résistance des bobines dans cette étude générale.

Les résultats de l'optimisation montrent que les formes optimales de courant d'alimentation dépendent des caractéristiques de machines et des connexions des phases, mais les tensions correspondantes présentent toujours une forme en créneaux [3]. Deux exemples sont donnés sur la figure 7. Sans connexion électrique la tension optimale est de la forme carrée (type $180^{\circ}$ ). Pour les connexions en triangle et en étoile, la forme optimale en créneaux de tension est du type $120^{\circ}$, car la somme de trois tensions composées doit être nulle dans ces cas.

Les recherches de la forme optimale de courant ont donc conduit à l'alimentation classique en créneau de tension. Mais l'intérêt de cette optimisation est que l'on sait maintenant que cette alimentation minimise le facteur de dimensionnement; elle est optimale. De plus, elle est simple à réaliser. On peut

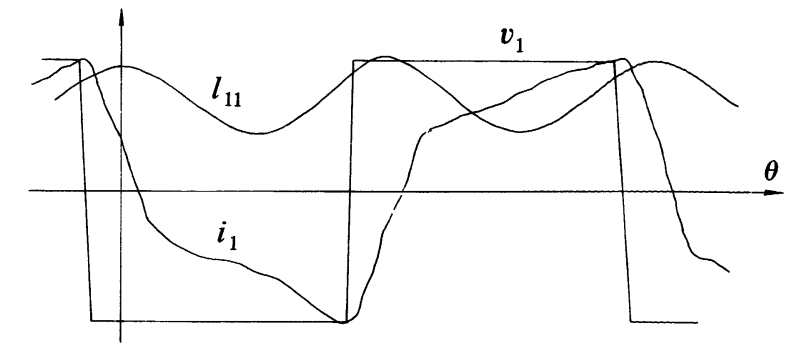

(a) Sans connexion électrique - $(L=1, \Delta L=0,5, M=$ $-0,3, \Delta M=0,5)$.

(a)

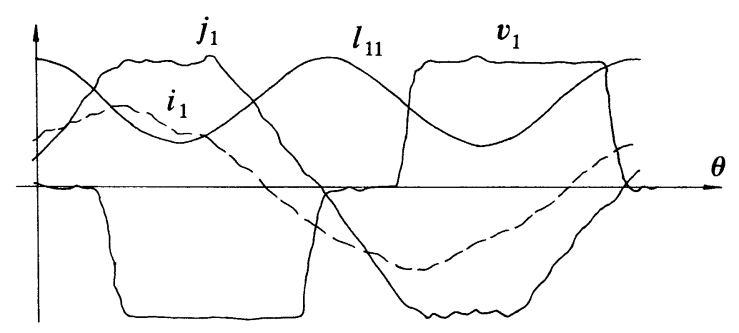

(b) En triangle - $(L=1, \Delta L=0,5, M=-0,3, \Delta M=$ $0,5)$.

(b)

Fig. 7. - Les formes optimales de courant et de tension d'alimentation.

[Optimal feeding current and voltage waveforms (a : without electric connection) (b : delta connection).]

l'obtenir avec les convertisseurs de la figure 4 sans utilisation de la technique PWM.

\section{Alimentation en créneau de tension.}

Quand la stratégie de l'alimentation en créneau de tension est choisie, deux variables déterminent complètement une telle forme en créneau : l'amplitude du créneau $u_{\max }$ et le déphasage entre la tension et l'inductance $\psi$. Nous pouvons maintenant écrire un nouveau programme moins complexe qui calcule les caractéristiques de fonctionnement des M.R.V. triphasées à partir de la tension en créneau imposée ( $u_{\max }$ et $\psi$ imposés). Nous pouvons ainsi étudier les influences de la fréquence d'alimentation, des connexions électriques, de l'excitation et des caractéristiques magnétiques de machine dans l'alimentation en créneau de tension [3]. Pour les différents cas, nous présentons, sur les tableaux I et II, les facteurs de dimensionnement optimaux et les ondulations relatives du couple obtenus respectivement pour les deux M.R.V. triphasées choisies : Machine Sans Mutuelle $(L=1, \Delta L=0,5, M=\Delta M=0)$ et Machine Avec Mutuelles $(L=1, \Delta L=0,5, M=$ $-0,3, \Delta M=0,5)$.

L'ondulation relative du couple qui constitue un 
Tableau I. - Comparaison des différents cas d'alimentation en créneau de tension pour Machine Sans Mutuelle.

[Comparison of different rectangular voltage feeding cases for Machine Without Mutuel.]

\begin{tabular}{|c|c|c|c|c|}
\hline \multirow{2}{*}{$\begin{array}{l}\text { Fréquence } \\
\text { Excitation }\end{array}$} & \multicolumn{2}{|c|}{$f_{i}=f_{l}$} & \multicolumn{2}{|c|}{$f_{m}=f_{l} / 2$} \\
\hline & $I_{0}=0$ & $I_{0}=1, \alpha=0,2$ & $I_{0}=0$ & $I_{0}=1, \alpha=0,2$ \\
\hline $\begin{array}{l}\text { Sans connexion } \\
\text { (unidirectionnel en courant) }\end{array}$ & $\begin{aligned} \delta & =5,93 \\
\delta c & =0,63\end{aligned}$ & $\begin{array}{r}\delta=4,53 \\
\delta c=0,73\end{array}$ & $\begin{array}{r}\delta=15,88 \\
\delta c=10,24\end{array}$ & $\begin{array}{r}\delta=17,67 \\
\delta c=13,90\end{array}$ \\
\hline $\begin{array}{l}\text { Sans connexion } \\
\text { (bidirectionnel en courant) }\end{array}$ & $\langle c\rangle=0$ & $\begin{aligned} \delta & =5,69 \\
\delta c & =1,17\end{aligned}$ & $\begin{aligned} \delta & =5,93 \\
\delta c & =0,63\end{aligned}$ & $\begin{array}{c}\delta=7,24 \\
\delta c=2,57\end{array}$ \\
\hline $\begin{array}{l}\text { Connexion } \\
\text { en triangle }\end{array}$ & $\langle c\rangle=0$ & $\begin{aligned} \delta & =9,07 \\
\delta c & =0,64\end{aligned}$ & $\begin{array}{r}\delta=12,33 \\
\delta c=1,32\end{array}$ & $\begin{aligned} \delta & =15,43 \\
\delta c & =3,97\end{aligned}$ \\
\hline $\begin{array}{l}\text { Connexion } \\
\text { en étoile }\end{array}$ & $\langle c\rangle=0$ & $\begin{aligned} \delta & =6,43 \\
\delta c & =0,69\end{aligned}$ & $\begin{array}{r}\delta=13,31 \\
\delta c=0,30\end{array}$ & $\begin{aligned} \delta & =18,41 \\
\delta c & =6,62\end{aligned}$ \\
\hline
\end{tabular}

Tableau II. - Comparaison des différents cas d'alimentation en créneau de tension pour Machine Avec Mutuelles.

[Comparison of different rectangular voltage feeding cases for Machine With Mutuels.]

\begin{tabular}{lcccc}
\hline \multicolumn{1}{c}{ Fréquence } & \multicolumn{2}{c}{$f_{i}=f_{l}$} & \multicolumn{2}{c}{$f_{m}=f_{l} / 2$} \\
\hline \multicolumn{1}{c}{ Excitation } & $I_{0}=0$ & $I_{0}=1, \alpha=0,2$ & $I_{0}=0$ & $I_{0}=1, \alpha=0,2$ \\
\hline $\begin{array}{l}\text { Sans connexion } \\
\text { (unidirectionnel en courant) }\end{array}$ & $\langle c\rangle=0$ & $\langle c\rangle=0$ & $\begin{array}{r}\delta=6,75 \\
\delta c=0,30\end{array}$ & $\begin{array}{r}\delta=6,96 \\
\delta c=0,30\end{array}$ \\
\hline $\begin{array}{l}\text { Sans connexion } \\
\text { (bidirectionnel en courant) }\end{array}$ & $\langle c\rangle=0$ & $\langle c\rangle=0$ & $\begin{array}{r}\delta=3,38 \\
\delta c=0,30\end{array}$ & $\begin{array}{r}\delta=3,58 \\
\delta c=0,30\end{array}$ \\
\hline $\begin{array}{l}\text { Connexion } \\
\text { en triangle }\end{array}$ & $\langle c\rangle=0$ & $\langle c\rangle=0$ & $\begin{array}{r}\delta=5,41 \\
\delta c=0,30\end{array}$ & $\begin{array}{r}\delta=5,72 \\
\delta c=0,30\end{array}$ \\
\hline $\begin{array}{l}\text { Connexion } \\
\text { en étoile }\end{array}$ & $\langle c\rangle=0$ & $\langle c\rangle=0$ & $\begin{array}{r}\delta=5,41 \\
\delta c=0,30\end{array}$ & $\begin{array}{r}\delta=6,33 \\
\delta c=0,30\end{array}$ \\
\hline
\end{tabular}

critère important pour les contraintes mécaniques et les vibrations est définie par :

$$
\delta c=\frac{c_{\max }-c_{\min }}{\langle c\rangle}
$$

où $\langle c\rangle$ désigne le couple moyen et $c_{\max }, c_{\min }$ sont respectivement la valeur maximale et la valeur minimale du couple instantané.

Observons le tableau I pour la Machine Sans Mutuelle, on voit que les connexions en triangle et en étoile présentent des facteurs de dimensionnement bien plus importants que ceux obtenus dans le cas sans connexion électrique. Sans excitation, l'alimentation à fréquence $f_{i}=f_{l}$ avec le convertisseur unidirectionnel en courant a le même facteur de dimensionnement et la même ondulation du couple que l'alimentation à fréquence $f_{m}=f_{l} / 2$ avec le convertisseur bidirectionnel dans le cas des trois phases non connectées. Nous préférons évidemment le montage unidirectionnel puisqu'il n'exige que la moitié de composants semi-conducteurs par rapport au montage bidirectionnel (Sect. 4.3). Si la machine possède un enroulement d'excitation, le facteur de dimensionnement peut encore être amélioré avec un courant d'excitation moins « coûteux » $(\alpha=0,2)$ 
mais l'ondulation du couple instantané sera augmentée.

Pour la Machine Avec Mutuelles (Tab. II), le convertisseur unidirectionnel dans le cas de trois phases non connectées n'a plus d'intérêt et l'alimentation à fréquence $f_{i}=f_{l}$ donne toujours un couple moyen nul. Le meilleur facteur de dimensionnement est obtenu dans le cas des machines à trois phases non connectées alimentées à fréquence $f_{m}=f_{l} / 2$ par le convertisseur bidirectionnel. Mais comme il exige deux fois plus de composants semi-conducteurs, c'est plutôt le cas des connexions en triangle ou en étoile qui minimise le dimensionnement du convertisseur. Dans l'alimentation à fréquence $f_{m}=f_{l} / 2$, l'excitation ne peut que détériorer les performances de la machine.

Les connexions en triangle et en étoile donnent exactement le même facteur de dimensionnement. Cependant, avec les mêmes enroulements, le convertisseur utilisé avec une machine connectée en triangle subit plus de contraintes en courant et celui utilisé avec une machine connectée en étoile plus de contraintes en tension. On peut bien sûr faire un choix de minimisation de la tension ou du courant en choisissant convenablement le nombre de spires des enroulements au moment de la conception de machine. On remarque enfin que l'ondulation relative du couple est toujours faible dans tous les cas d'alimentation pour la Machine Avec Mutuelles.

Nous avons aussi calculé les caractéristiques de fonctionnement pour des machines de caractéristiques différentes [3]. La figure 8 montre l'évolution
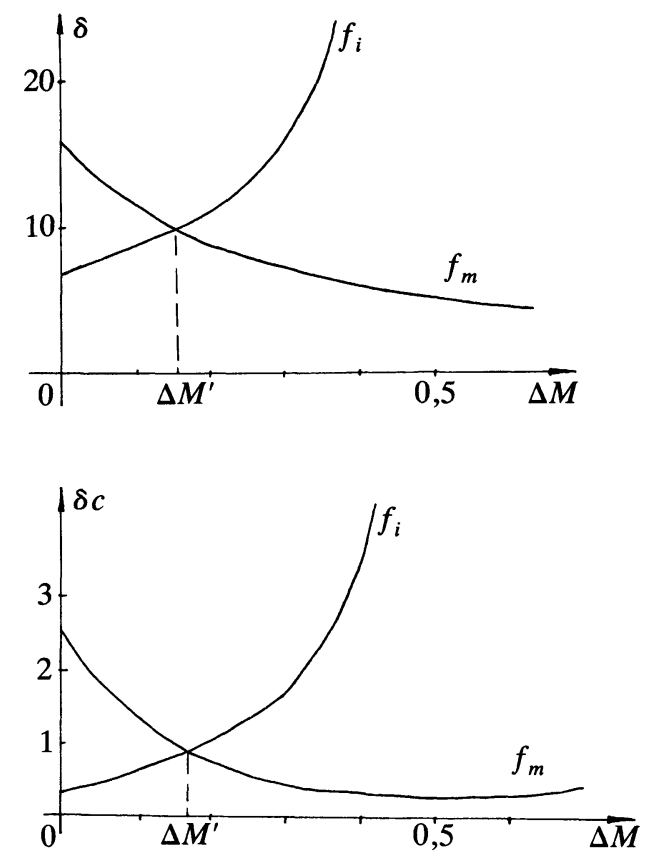

Fig. 8. - Evolution de $\delta$ et de $\delta c$ en fonction de $\Delta M$.

[Evolution of $\delta$ and $\delta c$ as a function of $\Delta M$.] du facteur de dimensionnement et de l'ondulation du couple en fonction de la variation des mutuelles $\Delta M$. Ces évolutions en fonction de $\Delta M$ sont tout à fait différentes pour les deux fréquences d'alimentation. On voit qu'il existe une valeur $\Delta M^{\prime}$ de $\Delta M$, en deçà de laquelle l'alimentation à fréquence $f_{i}=f_{l}$ donne le meilleur facteur de dimensionnement et au-delà de laquelle c'est l'alimentation à fréquence $f_{m}=f_{l} / 2$ qui est la plus intéressante.

\section{Influence de la fréquence d'alimentation.}

Nous avons déjà remarqué (Tab. II) que la Machine Avec Mutuelles alimentée à fréquence $f_{i}=f_{l}$ donne toujours un couple moyen nul. Nous allons expliquer d'une façon plus physique ce résultat $a$ priori peu évident. Pour faciliter le raisonnement, nous choisissons une alimentation en créneaux de courant. Mais le raisonnement est identique avec une forme sinusoïdale de courant ou une forme de courant correspondant à l'alimentation en créneaux de tension.

En raison de la symétrie des trois phases et grâce aux équations (3) et (4), on peut écrire l'expression $\mathrm{du}$ couple sous une forme liée à la phase 1 :

$$
c_{1}=\frac{1}{2}\left(i_{1}^{2} \frac{\mathrm{d} l_{11}}{\mathrm{~d} \theta}+i_{1} i_{2} \frac{\mathrm{d} l_{12}}{\mathrm{~d} \theta}+i_{1} i_{3} \frac{\mathrm{d} l_{13}}{\mathrm{~d} \theta}\right) \text {. }
$$

Pour l'alimentation à fréquence $f_{i}=f_{l}$, les formes des inductances, des courants et du couple lié à la phase 1 sont présentées sur la figure 9. La surface $A$ représente le couple moyen dû à l'inductance propre, donc le couple moyen en monophasé. Les surfaces $B$ et $C$ représentent les couples moyens générés par les mutuelles. L'interaction des courants des deux phases a lieu au moment de la décroissance de leurs mutuelles $\left(\mathrm{d} l_{i j} / \mathrm{d} \theta<0\right)$, le couple moyen dû aux mutuelles est donc négatif. Quand $\Delta L=\Delta M$, la surface $A$ est égale à la somme des surfaces $B$ et $C$. Il en résulte donc $\langle c\rangle=0$.

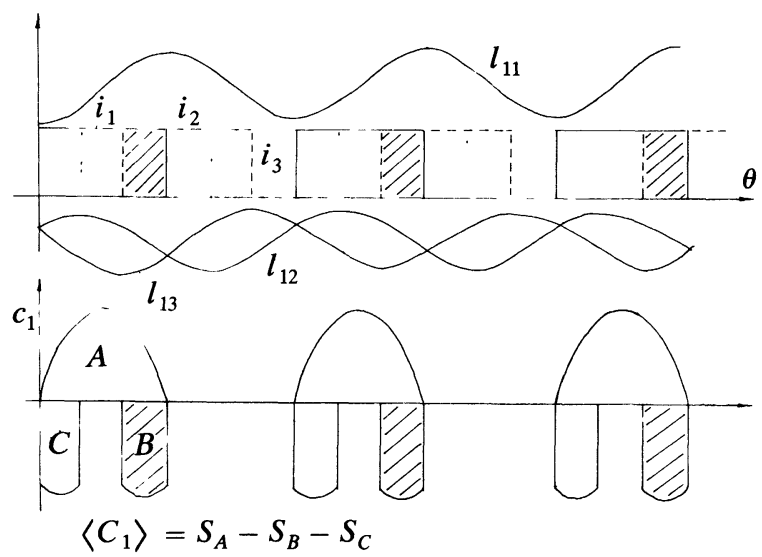

Fig. 9. - Couple des machines avec mutuelles alimentées à fréquence $f_{i}=f_{l}$.

[Torque of machine with mutual inductance and with feeding frequency $f_{i}=f_{l}$.] 
Pour l'alimentation à fréquence $f_{m}=f_{l} / 2$, la même démarche est présentée sur la figure 10. L'interaction des courants des deux phases a lieu toujours pendant la décroissance de leurs mutuelles, mais le produit des 2 courants est négatif, le couple moyen généré par les mutuelles est donc positif.
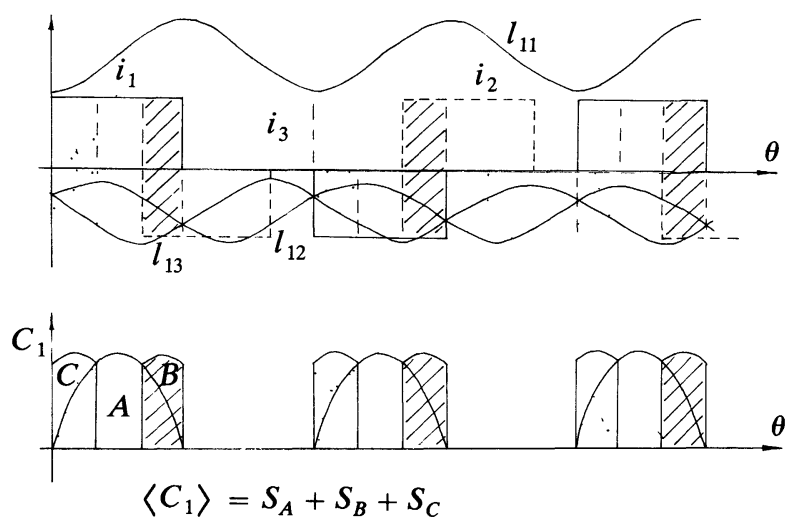

Fig. 10. - Couple des machines avec mutuelles alimentées à fréquence $f_{m}=f_{l} / 2$.

[Torque of machine with mutual inductance and with feeding frequency $f_{m}=f_{l} / 2$.]

Nous avons donc montré que les variations des mutuelles peuvent contribuer au couple moyen à condition d'alimenter la machine à la fréquence égale à la moitié de celle de l'inductance.

\section{Expérimentation.}

Pour vérifier expérimentalement nos résultats de simulation numérique, nous avons réalisé un montage d'essais avec une machine à réluctance variable triphasée avec mutuelles [3]. Les trois phases sont connectées en étoile et alimentées en créneau de tension par un convertisseur triphasé. La machine fonctionne en autopilotage. Le synoptique du montage est donné à la figure 11 .

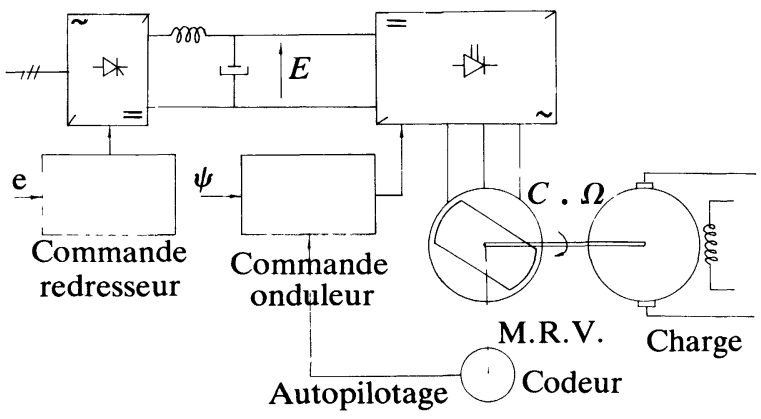

Fig. 11. - Synoptique du montage d'essais.

[Synoptic of test group.]
La comparaison entre mesures et simulations montre une très bonne concordance dans notre hypothèse du circuit magnétique non saturé. Ceci permet de vérifier la validité du modèle linéaire choisi et les programmes de la simulation. Nous donnons un exemple des courbes de courant et de tensions à la figure 12 .

$$
u_{\max }=E=172 \mathrm{~V}, \quad \psi=132^{\circ}, \quad N=1320 \mathrm{tr} / \mathrm{m}
$$
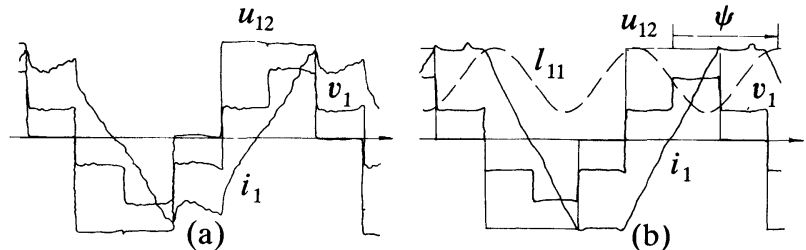

Fig. 12. - Formes de tensions et de courant (a : mesure et $\mathrm{b}:$ simulation).

[Voltage and current waveforms (a : measure and $b$ : simulation).]

L'angle $\psi$ est le déphasage entre l'inductance et le fondamental de la tension imposée. Cet angle $\psi$ et l'amplitude de tension $E$ constituent les deux seuls paramètres de réglage dans l'alimentation en créneaux de tension. La figure 13 montre l'influence de l'angle $\psi$ sur le couple moyen et le facteur de dimensionnement. On remarque que le facteur de dimensionnement minimal ne se trouve pas pour le même angle de déphasage $\psi$ que le couple moyen maximal $\left(\psi_{\mathrm{op}}<\psi_{M}\right)$. Mais l'allure de $\delta$ présente une zone assez « plate », une variation d'environ $20^{\circ}$ de $\psi$ autour du point optimal ne dégradera pas beaucoup le facteur de dimensionnement.
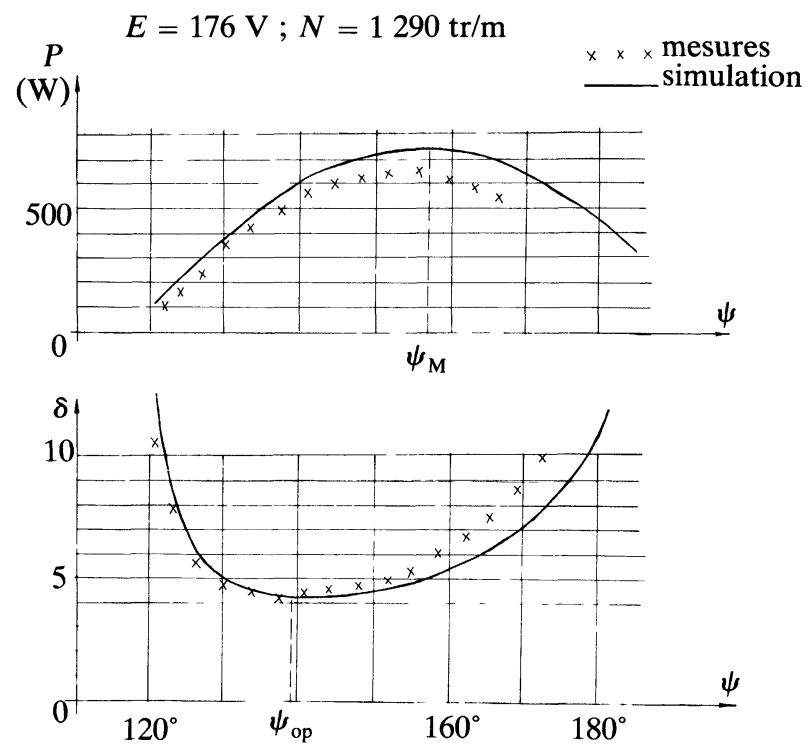

Fig. 13. - Evolution de $P$ et de $\delta$ en fonction de l'angle $\psi$. [Evolution of $P$ and $\delta$ as a function of the angle $\psi$.] 
Nous remarquons que les écarts sont d'autant plus importants que l'angle $\psi$ est plus grand. Ceci est principalement dû à notre modèle de machine qui ne prend pas en compte la saturation. Or le courant augmente en fonction de $\psi$. A partir de $\psi=$ $140^{\circ}$, la machine commence à être saturée et les écarts augmentent.

Si on fixe les 2 paramètres de réglage $\psi$ et $E$, et on fait varier la charge, on obtient l'évolution du couple moyen et du facteur de dimensionnement en fonction de la vitesse (Fig. 14).

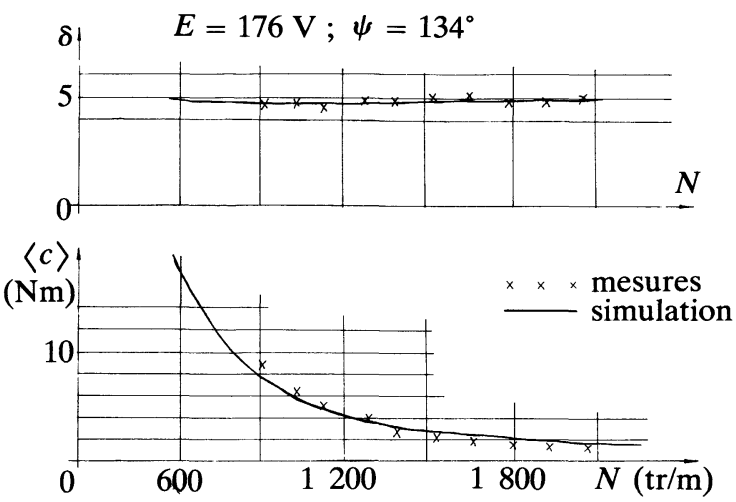

Fig. 14. - Evolution de $\delta$ et de $\langle c\rangle$ en fonction de la vitesse de machine.

[Evolution of $\delta$ and $\langle c\rangle$ as a function of the machine speed.]

On constate que la caractéristique mécanique naturelle $(\langle c\rangle$ en fonction de $N)$ de la M.R.V. autopilotée ressemble au type « Machine à courant continu à excitation série » : le couple décroît quand la vitesse augmente. Avec une commande électronique, on peut tout de même imposer un fonctionnement à vitesse constante ou à couple constant.

\section{Modes de commande optimale.}

Pour une application en vitesse variable, nous minimisons le facteur de dimensionnement et donc la taille du convertisseur au point nominal de fonctionnement de la machine $\left(\Omega_{N},\langle c\rangle_{N}\right)$. Mais pour des puissances inférieures, il n'est pas toujours nécessaire de minimiser encore le facteur de dimensionnement. Dans ce cas là, il est plutôt intéressant de minimiser l'ondulation du couple ou de maximiser le rendement de l'ensemble convertisseur-machine tout en s'assurant que le convertisseur fonctionne toujours en deçà des limites de tension et de courant préalablement choisies pour le point nominal. On pourrait effectuer une autre optimisation des formes de courant et de tension d'alimentation avec de nouveaux critères. Il est cependant plus convenable d'utiliser encore les mêmes formes de tension en créneaux pour conserver le même convertisseur et la même commande en optimisant les deux paramètres $E$ et $\psi$.

A la figure 15, nous présentons sur le plan couplevitesse $(\langle c\rangle, \Omega)$ les limites de fonctionnement et les critères d'optimisation pour les différents points de fonctionnement.

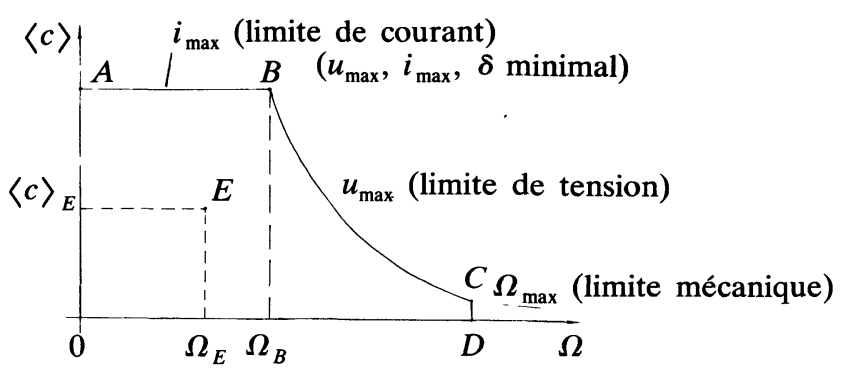

Fig. 15. - Limites de fonctionnement par le convertisseur.

[Working limits imposed by the converter.]

point B : puissance maximale. C'est en ce point qu'on cherche à optimiser le facteur de dimensionnement. Le convertisseur est ainsi dimensionné pour $u_{\max }$ et $i_{\max }$ qui limitent, par la suite, la tension et le courant dans tous les fonctionnements à puissance inférieure ;

courbe BC : limitation par la tension maximale $u_{\max }$. Avec $u_{\max }$ fixée, on cherche pour chaque $\Omega$ l'angle $\psi$ qui donne le couple maximal dans la condition $i \leqslant i_{\max }$;

courbe AB : limitation par le courant maximal $i_{\max }$. Le couple est directement lié au courant. Pour $i_{\max }$ donné, le couple doit être constant en fonction de $\Omega$. Quand la résistance des enroulements est négligée, le rapport $U / \Omega$ est constant sur cette droite ;

point $\mathrm{E}$ : un point quelconque dans les limites de fonctionnement. On cherche les deux paramètres de réglage $(E$ et $\psi)$ optimisant les performances de la machine (ex. : minimisant les pertes ou l'ondulation du couple). On doit surveiller que le courant et la tension ne dépassent pas leurs limites maximales $\left(i \leqslant i_{\max }\right.$ et $\left.u \leqslant u_{\max }\right)$.

Par cette considération, on pourra établir théoriquement une loi de commande en optimisant les performances de l'ensemble convertisseur-machine pour tous les points de fonctionnement possibles. On doit préalablement effectuer une optimisation et déterminer les paramètres de commande pour cha- 
que point de fonctionnement. La machine peut donc toujours travailler dans les conditions optimales sans dépasser les limites de tension et de courant de convertisseur.

\section{Conclusion.}

A partir d'une optimisation de la forme de courant d'alimentation, nous avons montré que c'est l'alimentation en créneaux de tension qui minimise le facteur de dimensionnement du convertisseur. Ceci est vrai pour les machines avec mutuelles comme pour les machines sans mutuelle. Cette alimentation est déjà classiquement utilisée. L'intérêt de cette étude est de montrer qu'elle est la meilleure au point de vue du dimensionnement du convertisseur d'alimentation.

Les machines sans mutuelles (machines multimonophasées) présentent des caractéristiques intéressantes. Le meilleur facteur de dimensionnement et une petite ondulation du couple sont obtenus dans le cas où les trois phases sont alimentées indépendam- ment par un convertisseur unidirectionnel à la fréquence de l'inductance. L'excitation peut, dans ce cas là, améliorer le facteur de dimensionnement, mais elle augmente en même temps l'ondulation du couple.

Cependant, les machines avec mutuelles peuvent encore améliorer les performances parce que les mutuelles peuvent contribuer au couple moyen. Elles diminuent le facteur de dimensionnement et l'ondulation du couple. Elles assurent très souvent une meilleure utilisation du circuit magnétique. Pour les machines avec mutuelles, il faut connecter les trois phases en étoile ou en triangle et alimenter la machine à la fréquence égale à la moitié de celle de l'inductance. L'excitation est dans ce cas là inutile.

Dans l'avenir, l'optimisation du facteur de dimensionnement pourrait être étendue à tous les points de fonctionnement. Il serait alors intéressant d'établir une loi de commande permettant de minimiser les pertes et l'ondulation du couple. La machine travaillerait ainsi toujours dans les meilleures conditions.

\section{Bibliographie}

[1] Glaize, C., « Recherche des formes optimales d'alimentation des machines à réluctance variable », Revue Phys. Appl. 20 (1985) 779-794.

[2] Multon, B., Glaize, C., «Optimisation du dimensionnement des alimentations des machines à réluctance variable ", Revue Phys. Appl. 22 (1987) 339-357.

[3] TIAN, X., «Etude des modes d'alimentation des machines à réluctance variable à phases magnétiquement couplées ", Thèse de doctorat de l’Université Paris VI (5 mai 1987).

[4] BARRET, Ph., Régimes transitoires des machines tournantes électriques (Eyrolles, Paris) 1982.

[5] FAucher, J., "Contribution à l'étude des machines à réluctance variable à commutation électronique ». Thèse d'Etat Toulouse (26 juin 1981).

[6] Davis, R. M., RAY, W. F., Blake, B. J., « Inverter drive for switched reluctance motors, circuit and component ratings », Proc. IEE, 128 (1981) 126136.
[7] Ray, W. F., Lawrenson, P. J., Davis, R. M. Stephenson, J. M., Fulton, N. N., Blake, R. J., " High-performance switched reluctance brushless drives », IEEE Trans. IA-22 (1986) $722-730$.

[8] Harris, M. R., Finch, J. W., Mallick, J. A., Miller, T. J. E., «A review of the integralhorsepower switched reluctance drive », IEEE Trans. IA-22 (1986) 716-721.

[9] Glaize, C., «Etude générale et optimisation des modes d'alimentation des machines à réluctance variable. Application aux machines polydiscoïdes ». Thèse d'Etat Paris VI (13 décembre 1983).

[10] Multon, B., «Contribution à l'optimisation des ensembles convertisseurs-machines à réluctance variable. Minimisation du facteur de dimensionnement ». Thèse de $3^{\mathrm{e}}$ cycle Paris VI ( 2 juillet 1985). 